\title{
Differences in Expression of Acetylcholinesterase and Collagen Q Control the Distribution and Oligomerization of the Collagen-Tailed Forms in Fast and Slow Muscles
}

\author{
Eric Krejci,, ${ }^{1}$ Claire Legay, ${ }^{1}$ Sébastien Thomine, ${ }^{1}$ Janez Sketelj, ${ }^{2}$ and Jean Massoulié ${ }^{1}$ \\ ${ }^{1}$ Laboratoire de Neurobiologie Cellulaire et Moléculaire, Centre National de la Recherche Scientifique, Unité Mixte de \\ Recherche 8544, Ecole Normale Supérieure, 75005 Paris, France, and 2/nstitute of Pathophysiology, School of Medicine, \\ University of Ljubljana, 1000 Ljubljana, Slovenia
}

\begin{abstract}
The collagen-tailed forms of acetylcholinesterase (AChE) are accumulated at mammalian neuromuscular junctions. The $A_{4}$, $A_{8}$, and $A_{12}$ forms are expressed differently in the rat fast and slow muscles; the sternomastoid muscle contains essentially the $A_{12}$ form at end plates, whereas the soleus muscle also contains extrajunctional $A_{4}$ and $A_{8}$ forms. We show that collagen $\mathrm{Q}(\mathrm{Col} \mathrm{Q})$ transcripts become exclusively junctional in the adult sternomastoid but remain uniformly expressed in the soleus. By coinjecting Xenopus oocytes with $\mathrm{AChE}_{\mathrm{T}}$ and $\mathrm{ColQ}$
\end{abstract}

At the vertebrate neuromuscular junction, acetylcholinesterase (AChE) (EC 3.1.1.7) hydrolyzes released acetylcholine, thereby controlling the duration of receptor activation. To serve this key function, $\mathrm{AChE}$ is highly concentrated at the neuromuscular junction. Mammalian muscles exclusively produce catalytic subunits of type $\mathrm{T}\left(\mathrm{AChE}_{\mathrm{T}}\right)$, which generate various oligomers (for review, see Massoulié et al., 1993). The collagen-tailed or asymmetric (A) forms are characterized by the presence of a specific collagen, ColQ (Krejci et al., 1991, 1997). In these molecules, each strand of the ColQ triple helix can be associated with a tetramer of $\mathrm{AChE}_{\mathrm{T}}$ subunits through its $\mathrm{N}$-terminal proline-rich attachment domain (PRAD) (Bon et al., 1997). The $\mathrm{A}_{4}, \mathrm{~A}_{8}$, and $\mathrm{A}_{12}$ forms contain, respectively one, two, and three $\mathrm{AChE}$ tetramers.

It has long been known that collagen-tailed AChE forms are accumulated at neuromuscular junctions (Hall and Kelly, 1971; Hall, 1973; Sketelj and Brzin, 1979) in the basal lamina, within the synaptic cleft of the neuromuscular junction. Inactivation of the ColQ gene in mice recently provided direct evidence for the role of these molecules in neuromuscular transmission (Feng et al., 1999); although the mice may live for several weeks, they show severe muscle dysfunction. Their neuromuscular junctions do not show any accumulation of AChE activity. A similar situation occurs in severely affected congenital myasthenic syndrome (CMS-1c) patients in whom a total lack of AChE accumulation at

\footnotetext{
Received June 15, 1999; revised Sept. 20, 1999; accepted Oct. 7, 1999.

This work was supported by grants from the Centre National de la Recherche Scientifique, the Association Française contre les Myopathies, the Direction des Forces et de la Prospective, the European Community, and the Ministry of Science and Technology of the Republic of Slovenia. We thank Monique Lambergeon and Boris Pecenko for expert technical assistance.

Correspondence should be addressed to Jean Massoulié, Laboratoire de Neurobiologie Cellulaire et Moléculaire, Centre National de la Recherche Scientifique Unité Mixte de Recherche 8544, Ecole Normale Supérieure, 46 rue d’Ulm, 75005 Paris, France. E-mail: jean.massoulie@biologie.ens.fr.

Copyright (C) 1999 Society for Neuroscience 0270-6474/99/1910672-08\$05.00/0
}

mRNAs, we reproduced the muscle patterns of collagen-tailed forms. The soleus contains transcripts ColQ1 and ColQ1a, whereas the sternomastoid only contains ColQ1a. Collagentailed AChE represents the first evidence that synaptic components involved in cholinergic transmission may be differently regulated in fast and slow muscles.

Key words: neuromuscular junction; acetylcholinesterase; collagen; fast muscle; slow muscle; rat end plates is caused by various mutations in the ColQ gene (Donger et al., 1998; Ohno et al., 1998).

In rat and mouse, numerous studies have shown that the expression and distribution of AChE forms in muscles depend on age, on the fast or slow type of muscle, and on activity (Sketelj and Brzin, 1980; Lømo et al., 1985; Toutant and Massoulié, 1988). During development, $\mathrm{AChE}_{\mathrm{T}}$ transcripts in muscle fibers accumulate beneath the synaptic contacts as soon as they are established (Legay et al., 1995). In rat, collagen-tailed forms appear simultaneously with nerve-muscle contacts (Vigny et al., 1976; Fernandez and Seiter, 1984). At birth, asymmetric forms are expressed all along the muscle fibers (Sketelj and Brzin, 1980; Koenig and Rieger, 1981; Fernandez and Seiter, 1984). Fast and slow muscles differentiate postnatally, in particular in their expression of AChE forms; in the adult, slow muscles contain less AChE activity than fast muscles, and A forms remain present in the extrajunctional domains of slow muscles, although they are restricted to the junctions of fast muscles (Lai et al., 1986; Čresnar et al., 1994). In addition, slow muscles contain a high proportion of $A_{8}$ and $A_{4}$ forms, whereas fast muscles express mainly the $A_{12}$ form of AChE. In this paper, we examine the mechanisms that control the muscle-specific expression of collagen-tailed AChE forms at junctional and extrajunctional sites.

\section{MATERIALS AND METHODS}

Materials. Unless otherwise indicated, reagents were purchased from Prolabo (Paris, France), Sigma (St. Louis, MO), or Appligene (Illkirch, France), and enzymes were from New England Biolabs (Ozyme, France).

Dissection of junctional and extrajunctional segments of muscles; RNA extraction. Sternomastoid muscles were isolated from a group of 10 male rats weighing 200-250 gm. The narrow region in which all the motor end plates are located was identified under the low-power magnification microscope and separated from the rest of the muscle by a special knife with double razor blades $2.5-3 \mathrm{~mm}$ apart (junctional region). Approximately 2-mm-long pieces of muscle adjacent to this region were discarded, and the rest of the muscle represented the regions devoid of motor end plates (the extrajunctional region). All muscle samples were 
SOL AChE

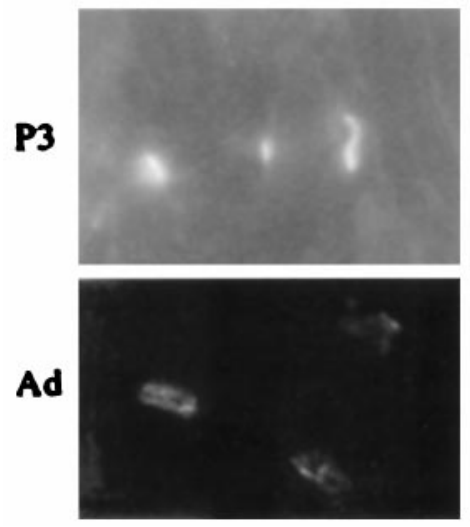

ColQ
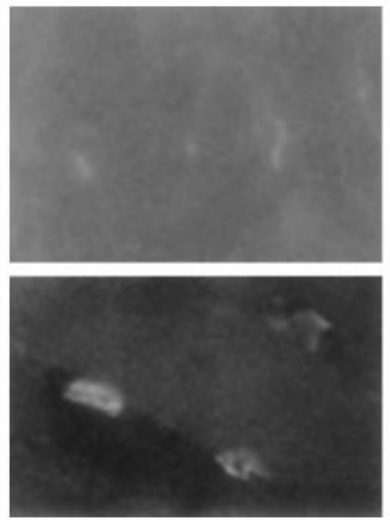

STM

AChE
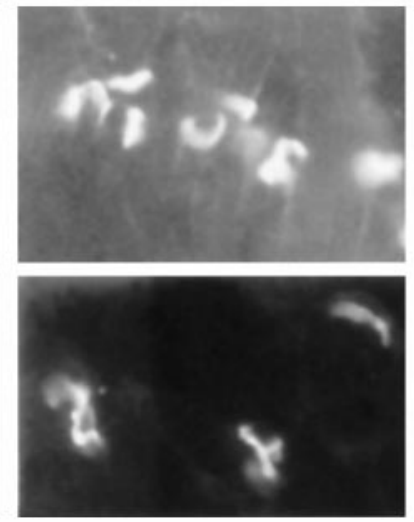

ColQ

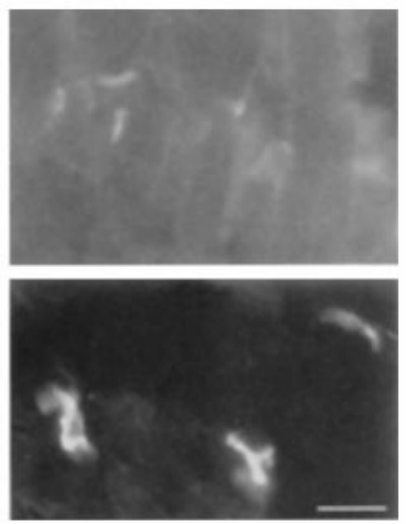

Figure 1. Immunofluorescence localization of AChE and ColQ proteins in the sternomastoid (STM) and soleus (SOL) muscles of newborn (P3) and adult $(A d)$ rats. Muscle sections were doubly stained with antibodies to AChE and ColQ. At both stages of development, the proteins colocalize in clusters. Scale bar: adult $(A d)$ muscles, $10 \mu \mathrm{m}$; newborn $(P 3)$ muscles, $5 \mu \mathrm{m}$.

immediately frozen in liquid nitrogen and kept at $-80^{\circ} \mathrm{C}$ until further analysis. Soleus muscles were isolated from the same rats. After visualizing the end plates by histochemical staining of AChE activity (Koelle and Friendenwald, 1949), junctional and extrajunctional regions were dissected and frozen in liquid nitrogen.

RNA was extracted by the single step method (Chomczynski and Sacchi, 1987). Whole muscles from 3 d old rat muscles were directly extracted in glass-glass Potter homogenizer, by the same method.

$R$ Nase protection assays. RNA probes were generated by synthesis with [ $\alpha^{32}$ P]UTP $(800 \mathrm{Ci} / \mathrm{mmol}$; Amersham Pharmacia Biotech, Uppsala, Sweden). For the glyceraldehyde 3-phosphate dehydrogenase (GAPDH) probe, $2.5 \mu \mathrm{l}$ of $\left[\alpha^{32} \mathrm{P}\right] \mathrm{UTP}$ was diluted with $1 \mu \mathrm{l}$ of $10 \mathrm{~mm} \mathrm{UTP}$, so that the specific radioactivity was reduced 160 -fold. The ColQ probe (see Fig. 3) corresponds to nucleotides (nt) 3-342 of rQ1, cloned in TA vector (Invitrogen, San Diego, CA), which was linearized with DdeI. The probe, synthesized with SP6 RNA polymerase, is $362 \mathrm{nt}$ long and protects a 268 nt long fragment of ColQ1 mRNA.

Specific probes for ColQ1 and ColQ1a (see Fig. 6) correspond respectively to fragments 46-258 (GenBank accession number AF 007583) and 3-258, cloned in the pCDNA3 vector (Invitrogen), which was linearized with HindIII. The probes, synthesized with SP6 RNA polymerase, are 300 and $280 \mathrm{nt}$ long and protect fragments of 210 and $190 \mathrm{nt}$ in their respective mRNAs. The AChE probe corresponds to part of the coding sequence 1592-1722 (GenBank accession number AF 550879). This probe, synthesized with SP6 RNA polymerase, is 220 long and protects a fragment of $130 \mathrm{nt}$.

The probes were gel purified and eluted in 2 mM EDTA and $0.5 \%$ SDS at $37^{\circ} \mathrm{C}$.

Hybridization of RNA with the radioactive probes, RNase inactivation, and digestion were performed with the RNase protection assays (RPA) High Speed kit (Ambion, Austin, TX). The protected fragments were separated by electrophoresis in nondenaturing gels and analyzed in a Fuji image analyzer (BAS 1000; Fuji, Tokyo, Japan), after $24 \mathrm{hr}$ exposure to the image plate. The gel was then exposed for 2 weeks to the film and scanned for image presentation. The content of A nucleotides was taken into account for calculating the abundance of the fragments, which were normalized to the GAPDH signal.

Injection of $m R N A$ into Xenopus oocytes. We constructed plasmids in which the $\mathrm{AChE}_{\mathrm{T}}$ and $\mathrm{ColQ}\left(\mathrm{rQ}_{1}\right)$ coding sequences were inserted between $5^{\prime}$ and $3^{\prime}$ untranslated sequences of Xenopus globin mRNA, so as to enhance stability and translation efficiency. The ColQ1 transcripts were deleted from their own $5^{\prime}$ untranslated sequence, which were replaced by $3 \mathrm{nt}$ from the ColQ1a $5^{\prime}$ untranslated sequence. Capped synthetic transcripts were prepared in vitro with MegaScript (Ambion). Aliquots of $\sim 50 \mathrm{nl}$ were injected into the animal poles of Xenopus oocytes. Analysis of AChE molecular forms was performed $20 \mathrm{hr}$ after injection.

Extraction of AChE and sedimentation analysis in sucrose gradients. $\mathrm{AChE}$ was extracted from Xenopus oocytes in $40 \mu \mathrm{l} /$ oocyte of ice-cold extraction solution (50 mM Tris- $\mathrm{HCl}$, $\mathrm{pH} 7,10 \mathrm{~mm} \mathrm{MgCl}_{2}, 0.8 \mathrm{M} \mathrm{NaCl}$, $1 \%$ Brij-96, $2 \mathrm{~mm}$ benzamidine, $20 \mu \mathrm{g} / \mathrm{ml}$ pepstatin, and $40 \mu \mathrm{g} / \mathrm{ml}$ leupeptin). The use of a high salt buffer allows the solubilization of collagen-tailed A forms, which aggregate at low ionic strength (Bon et al., 1978). The extracts were clarified by a short centrifugation that facilitates the determination of AChE activity by the method of Ellman et al. (1961).

Sedimentation analysis of AChE forms was performed in 5-20\% sucrose gradients containing $0.8 \mathrm{M} \mathrm{NaCl}, 50 \mathrm{~mm}$ Tris- $\mathrm{HCl}, \mathrm{pH} 7,10 \mathrm{~mm}$ $\mathrm{MgCl}_{2}$, and $1 \%$ Brij-96; they were centrifuged at $40,000 \mathrm{rpm}$ at $7^{\circ} \mathrm{C}$ for $16 \mathrm{hr}$, in a SW41 Beckman Instruments (Fullerton, CA) rotor. AChE was assayed by the colorimetric method of Ellman et al. (1961), by two successive readings to determine the reaction rate. The sedimentation coefficients were deduced by a linear relationship from the position of internal marker proteins alkaline phosphatase $(6.1 \mathrm{~S})$ and $\beta$-galactosidase (16 S) from Escherichia coli.

Immunohistochemistry. Fresh muscles were frozen and cut "en face" in a Leica (Nussloch, Germany) cryostat. The $10 \mu \mathrm{m}$ sections were fixed in $2 \%$ paraformaldehyde and incubated for $1 \mathrm{hr}$ with a polyclonal rat anti-AChE raised in rabbit (diluted 1: 400 in PBS-BSA) (Marsh et al., 1984), together with hen antibodies directed against a peptide corresponding to residues 35-51 of the $\mathrm{rQ}_{1}$ deduced primary sequence (Krejci et al., 1997). The tissues were then washed three times with PBS-BSA and incubated simultaneously with fluorescein-conjugated monoclonal antibodies to chicken light chains and tetramethylrhodamine-conjugated anti-rabbit IgG (both from The Jackson Laboratory, Bar Harbor, ME). After $1 \mathrm{hr}$ incubation with the secondary antibodies, the sections were rinsed three times in PBS and mounted in glycerol (Vectashield mounting medium; Vector Laboratories, Burlingame, CA).

In situ hybridization. In situ hybridizations were performed on wholemount muscles dissected from rats aged 4 weeks or postnatal day 3 (P3). The muscles were fixed overnight by immersion in $4 \%$ paraformaldehyde, and hybridizations were performed as described previously (Legay et al., 1995). The AChE riboprobe corresponds to an 800 bp fragment (coding sequence nt 879-1722); the ColQ riboprobe covers nt 182-856 of the ColQ1 coding sequence.

\section{RESULTS}

Synaptic accumulation of $A C h E$ and ColQ proteins and of their respective $m R N A$ s in the rat soleus and sternomastoid muscles

Using polyclonal antibodies raised against an $\mathrm{N}$-terminal peptide of ColQ (A17C) (Krejci et al., 1997) and against $\mathrm{AChE}_{\mathrm{T}}$ (A63) (Marsh et al., 1984), we examined the localization of the two proteins by immunofluorescence $3 \mathrm{~d}$ after birth and in the adult. Both proteins were found to be accumulated at the neuromuscu- 


\begin{abstract}
ColQ transcripts in whole-mount sternomastoid $(S T M)$ and soleus (SOL) muscles of newborn $(P 3)$ and adult rats. In newborn (P3) and adult ( $A d)$ muscles, AChE mRNAs are concentrated in the synaptic zone. ColQ mRNAs are diffuse along the muscle fibers, except in adult STM. Scale bar: adult STM and soleus hybridized with the ColQ probe, $15 \mu \mathrm{m}$; adult soleus and STM hybridized with the AChE probe and P3 soleus and STM hybridized with the ColQ probe, 12 $\mu \mathrm{m}$; P3 soleus and STM hybridized with the AChE probe, $6 \mu \mathrm{m}$.
\end{abstract}

SOL

AChE



ColQ



STM

AChE



ColQ

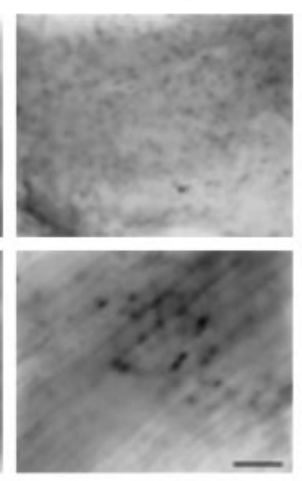

lar junctions in soleus and sternomastoid (STM) muscles at the newborn and adult stages (Fig. 1).

We then examined the distribution of AChE and ColQ mRNAs by in situ hybridization. For this purpose, we hybridized whole-mount muscles with riboprobes corresponding to the catalytic domain of $\mathrm{AChE}$ and to the N-terminal part of ColQ. As shown in Figure 2, AChE mRNAs are concentrated around junctional nuclei in all cases. In contrast, in situ hybridization with the ColQ probe produced a uniform signal in newborn muscles and in the adult soleus, illustrating a clear difference between gene expression and the accumulation of the mature protein product. An accumulation of ColQ transcripts around junctional nuclei could only be detected in the adult STM.

\section{Quantitative relationship between AChE and ColQ transcripts}

We analyzed the ColQ transcripts and AChE transcripts by RPA. We used a ColQ probe corresponding to exons 2, 3, and 4. This probe corresponds to the region encoding the PRAD in exon 2 and to the beginning of the collagen domain. In the same experiments, we also used a probe corresponding to AChE and a probe corresponding to GAPDH as an internal standard. These probes were hybridized with RNA samples extracted from junctional and extrajunctional segments of adult soleus and STM muscles (Fig. $3 A$ ). In agreement with in situ hybridization, we found that ColQ transcripts were present in the end plate and non-end plate regions of adult soleus, but only in the end plate region of adult STM. The absence of ColQ transcripts in the extrajunctional domain of the STM was confirmed by PCR analysis using specific primers (data not shown). In the STM, it is likely that ColQ transcripts are only produced by junctional nuclei; in the soleus, it is difficult to assess the contribution of these nuclei because of the presence of ColQ transcripts produced by the more numerous nonjunctional nuclei.

The relative amounts of ColQ and AChE mRNAs were quantified as indicated in Materials and Methods. AChE transcripts were present in all samples; they were markedly more abundant in the STM than in the soleus, as reported previously (Čresnar et al., 1994), and their level was slightly higher in end plate than in non-end plate regions of the adult muscles (Fig. $3 B$ ). In the soleus muscle, ColQ transcripts are uniformly distributed in the extrajunctional region, and the ratio of $\mathrm{AChE} / \mathrm{ColQ}$ mRNAs is $\sim 1.5$. In the STM muscle, it is possible to evaluate the specific contribution of the junctional nuclei in which both mRNAs are accumulated, as shown by in situ hybridization; by subtracting the
GAPDH-normalized mRNA content of the extrajunctional region from that of the junctional region, we find approximately eight copies of AChE mRNA per ColQ mRNA.

\section{Reconstitution of the muscle patterns of A forms by expression of AChE and ColQ mRNAs in different ratios in Xenopus oocytes}

Because fast and slow muscles seem to differ in the ratio of $\mathrm{AChE}$ and ColQ mRNAs, we thought that this might determine their different patterns of collagen-tailed AChE forms. In an attempt to reproduce the situation observed in muscles, we expressed defined mixtures of synthetic transcripts in Xenopus oocytes. In these experiments, the total AChE activity was proportional to the amount of $\mathrm{AChE}_{\mathrm{T}}$ transcripts. When $\mathrm{AChE}_{\mathrm{T}}$ was expressed without ColQ at a low level, it produced essentially monomers $\left(G_{1}\right)$ with a small proportion of dimers $\left(G_{2}\right)$; at a higher level of injected mRNA, we observed an additional 13.7 peak, corresponding to unstable aggregates as reported previously in COS cells (Bon and Massoulié, 1997).

Figure 4, $A$ and $B$, show the patterns of AChE forms obtained with the same amount of mRNA encoding ColQ1, injected together with different amounts of mRNA encoding $\mathrm{AChE}_{\mathrm{T}}$. At low $\mathrm{AChE}_{\mathrm{T}}$, we obtained the unsaturated $\mathrm{A}_{8}$ and $\mathrm{A}_{4}$ forms, with a low level of free $\mathrm{AChE}$ subunits, as in the extrajunctional domain of the mature soleus muscle. With a fourfold higher amount of $\mathrm{AChE}_{\mathrm{T}}$ mRNA, we obtained four times as much AChE activity, mostly as the saturated $\mathrm{A}_{12}$ form, together with a high level of free AChE subunits, as in the junctional domain of the STM muscle (Čresnar et al., 1994).

\section{Alternative transcription initiation sites produce two differentially expressed CoIQ transcripts, ColQ1 and ColQ1a}

When analyzing ColQ transcripts, we discovered the existence of two alternative exons corresponding to distinct transcription initiation sites. These exons, 1 and 1a, precede the PRAD-encoding exon 2. Figure $5 A$ shows the peptide and nucleotide sequences encoded by exons 1 and 1a; both seem to encode signal peptides, targeting the protein into the endoplasmic reticulum, so that the mature collagen chains begin with 13 residues encoded by exon 1 or 6 residues encoded by exon 1a. Thus, ColQ transcripts containing exons 1 and 1a encode collagen subunits that both possess a PRAD and can therefore organize tetramers. In the heart ventricle, ColQ transcripts are abundant, as shown previously 


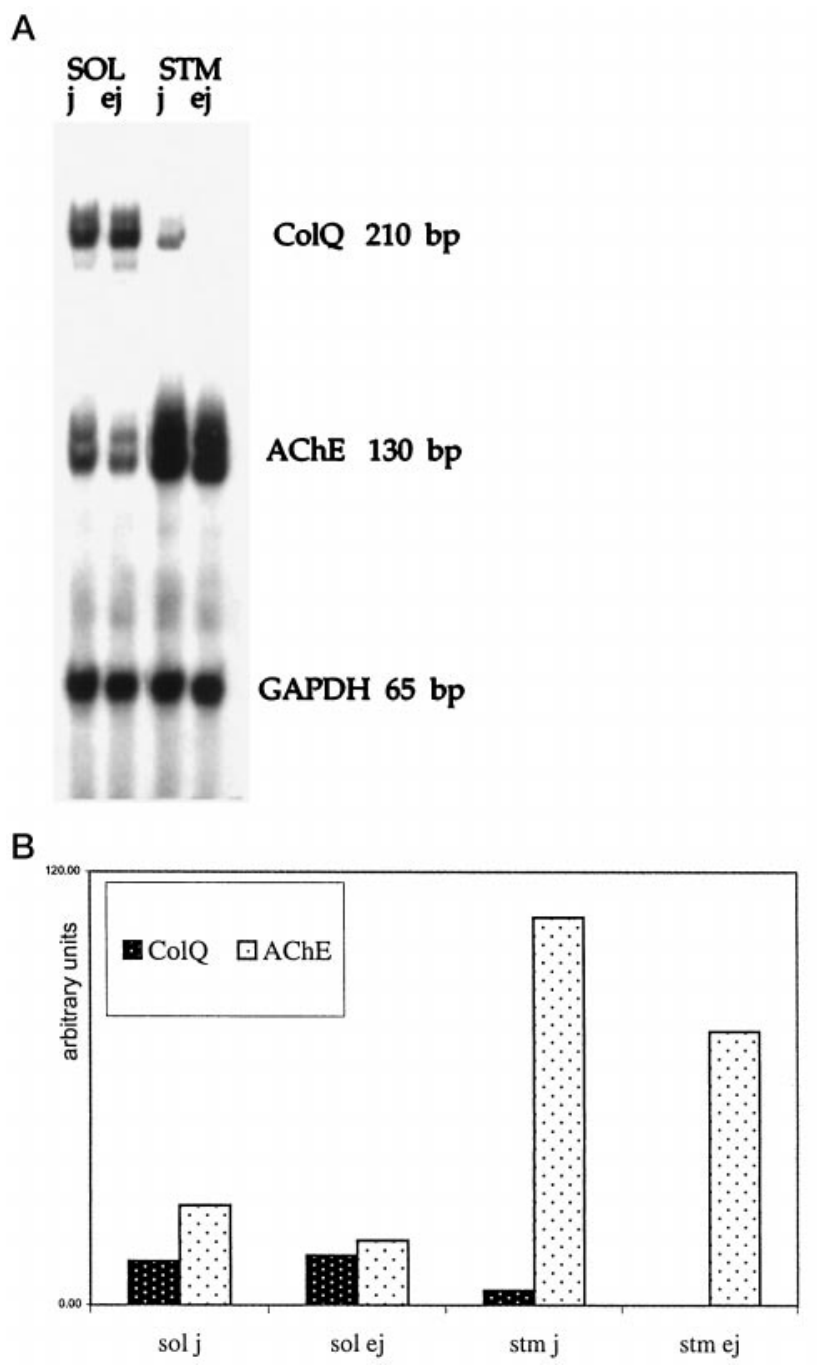

Figure 3. Quantitative relationship between $\mathrm{AChE}$ and ColQ transcripts in fast and slow muscles. $A$, RNase protection assay analysis: $15 \mu \mathrm{g}$ of total RNA from junctional $(j)$ and extrajunctional $(e j)$ domains of the soleus $(S O L)$ and sternomastoid (STM) muscles was hybridized with a ColQ probe covering the PRAD exon and the first part of the collagen domain, an AChE probe covering part of the catalytic domain, and a GAPDH probe, used as standard. After digestion with RNase, the protected fragments were separated in nondenaturing polyacrylamide gels that were then dried and exposed for 2 weeks to an autoradiographic film. To obtain comparable intensities, the specific radioactivity of the GAPDH probe was $1 / 160$ of that of the ColQ and AChE probes. $B$, Histogram representation of the relative abundance of the different transcripts, normalized to A content and GAPDH.

(Krejci et al., 1997), and correspond predominantly to ColQ1, with a barely detectable contribution of ColQ1a (Fig. $5 B$ ).

Using two probes corresponding to exon 1-exon 2 and to exon 1a-exon 2, we analyzed the proportions of ColQ1 and ColQ1a transcripts by RPA in soleus and STM muscles at the newborn and adult stages. As shown in Figure 6, we observed protected fragments corresponding to exon 1 -exon 2 or to exon $1 \mathrm{a}$-exon 2 , depending on the muscle. The levels of the two fragments indicate that the two transcripts are present at comparable levels in newborn fast muscles. Both transcripts were also detected in newborn soleus muscles, but the ColQ1a transcript prevailed. This is also true in the adult soleus. In the adult STM, however, the end plate-restricted ColQ transcripts exclusively contain exon
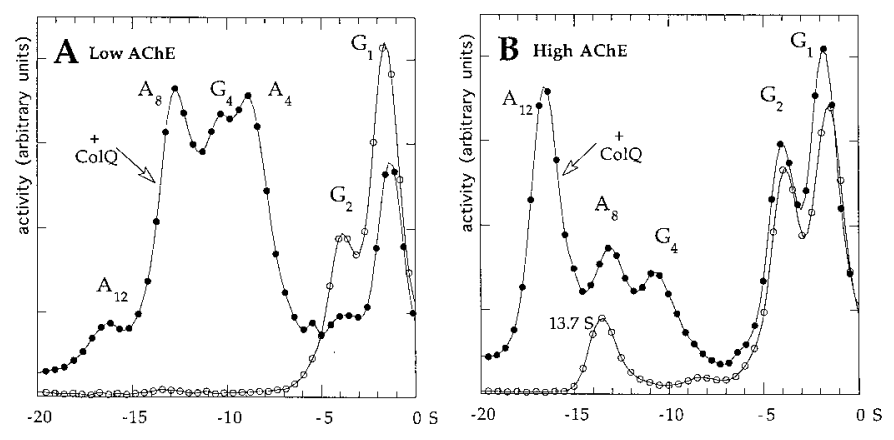

Figure 4. The pattern of collagen-tailed $\mathrm{A}_{12}, \mathrm{~A}_{8}$, and $\mathrm{A}_{4}$ is determined by the quantity of $\mathrm{AChE}_{\mathrm{T}}$. Xenopus oocytes were injected with a fixed amount of ColQ1 mRNA ( $0.25 \mathrm{ng} /$ oocyte) or with water (control), together with a low level of $\mathrm{AChE}_{\mathrm{T}}$ mRNA (0.62 ng/oocyte) $(A)$ or with a high level of $\mathrm{AChE}_{\mathrm{T}}$ mRNA (2.5 ng/oocyte) $(B)$. The activity is similar with or without ColQ and proportional to the amount of injected $\mathrm{AChE}$ mRNA. AChE oligomers were separated in $5-20 \%$ sucrose gradients containing salt and Brij 96, so that the $\mathrm{G}_{1}$ and $\mathrm{G}_{2}$ forms sediment at 2 and $3 \mathrm{~S}$. The identification of the oligomers is deduced from their position in the gradient relative to internal sedimentation markers. Note that the level of the $13.7 \mathrm{~S}$ oligomer depends on the level of $\mathrm{AChE}_{\mathrm{T}}$ expressed in the cell. The Xenopus oocytes did not produce any detectable level of tetrameric $\mathrm{G}_{4}$ form when expressing $\mathrm{AChE}_{\mathrm{T}}$ alone. A $10 \mathrm{~S} \mathrm{G}_{4}$ form appeared, however, when it was expressed with ColQ, indicating that this form was organized by the PRAD. These tetramers may either contain a complete ColQ subunit, which did not form a collagen trimer, or only its $\mathrm{N}$-terminal fragment.

1a (Fig. 6). This was confirmed by reverse transcription-PCR (data not shown).

\section{The ColQ1 and ColQ1a transcripts differ in the efficiency of assembly of collagen-tailed AChE forms in Xenopus oocytes}

Because the expression of ColQ1 and ColQ1a is specifically regulated during muscle differentiation, we asked whether they are equivalent in their association with $\mathrm{AChE}_{\mathrm{T}}$. We injected constant amounts of $\mathrm{AChE}_{\mathrm{T}}$ together with variable amounts of ColQ1 and ColQ1a mRNAs in oocytes. As shown in Figure 7, similar patterns of molecular forms were obtained with approximately two to four times more ColQ1 mRNA than ColQ1a mRNA. The ColQ1a transcripts, therefore, seem to be more efficient to trigger assembly of the catalytic AChE subunits into hetero-oligomers than the ColQ1 transcripts. This difference was not abolished when the short 5' untranslated sequences of ColQ1 and ColQ1a were replaced by the $5^{\prime}$ untranslated sequence of Xenopus globin, followed by three $5^{\prime}$ untranslated nucleotides of ColQ1a.

\section{DISCUSSION}

The ratio of ColQ and $A C h E_{T}$ subunits governs the production of $A_{12}, A_{8}$, and $A_{4}$ collagen-tailed forms

The collagen-tailed $\mathrm{A}_{12}$ molecular form of $\mathrm{AChE}$ contributes most of AChE activity in the mammalian neuromuscular junctions (Hall, 1973; Sketelj and Brzin, 1985). Recent genetic studies in mouse and human provided direct evidence that the collagentailed AChE molecules represent the functional enzyme species essential for normal neuromuscular transmission (Donger et al., 1998; Ohno et al., 1998; Feng et al., 1999). Knowledge about the mechanisms involved in assembly and accumulation of these AChE molecules in muscle fibers is, therefore, of great importance for understanding the development and function of the neuromuscular junction. 
exon 1

MAVLNPMTLGIYLQLFFCSIVSQPTFINSVLPISA

exon 1a

M M I G L S F S F T P L L L I V S G L L C H S A G Y W

exon 2

A L P G L Q K K R G N K A C C L L M P P P P P L P P P F F R S R P

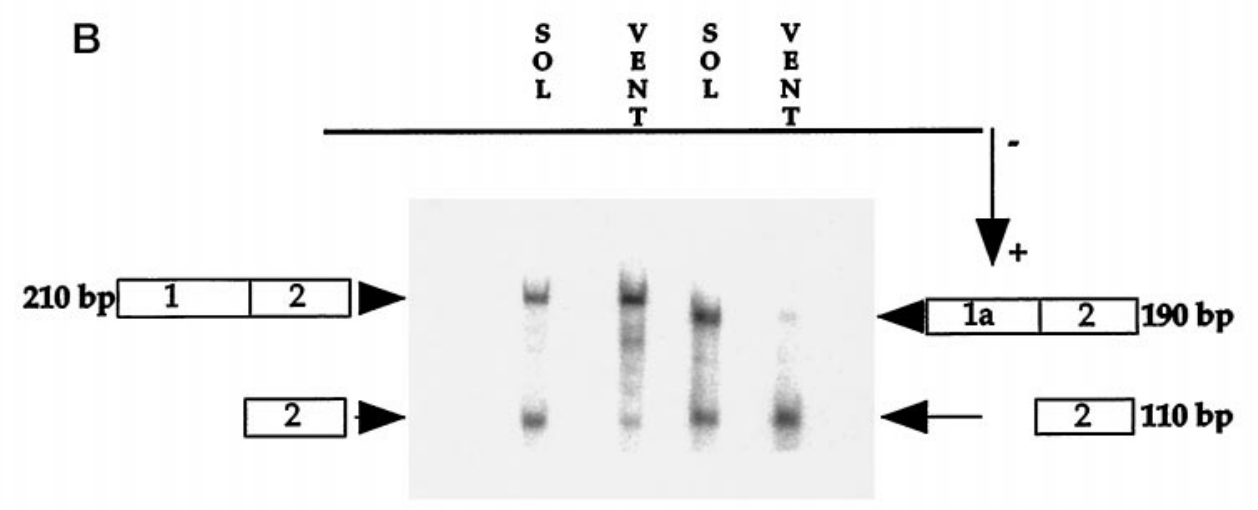

Figure 5. Alternative 5' exons of the ColQ gene. $A$, Peptide sequences encoded by rat exon 1, exon 1a, and exon 2. The predicted signal peptide is shown in bold type. $B$, Differential expression of the alternative initiation site variants: $15 \mu \mathrm{g}$ of RNA from soleus muscle $(S O L)$ and heart ventricle (VEN) was analyzed by RPA with two probes, covering alternative exons 1 or $1 \mathrm{a}$ and 2 . Exon 1 is the only variant found in ventricle, whereas exon $1 \mathrm{a}$ is the major variant in the soleus.

Figure 6. Quantitative relationship between ColQ1 and ColQ1a in fast and slow muscles. RPA analysis of the $5^{\prime}$ end of ColQ1a and ColQ1: $15 \mu \mathrm{g}$ of RNA from whole $3 \mathrm{~d}$ muscles (P3) and from adult $(A d)$ muscles [extrajunctional domain of soleus $(S O L)$ and junctional domain of sternomastoid muscle $(S T M)$ ] were hybridized with probes covering parts of exon 1a or exon 1 and exon 2, together with a GAPDH probe, used as standard. After digestion with RNase, the protected fragments were analyzed as in Figure 3.

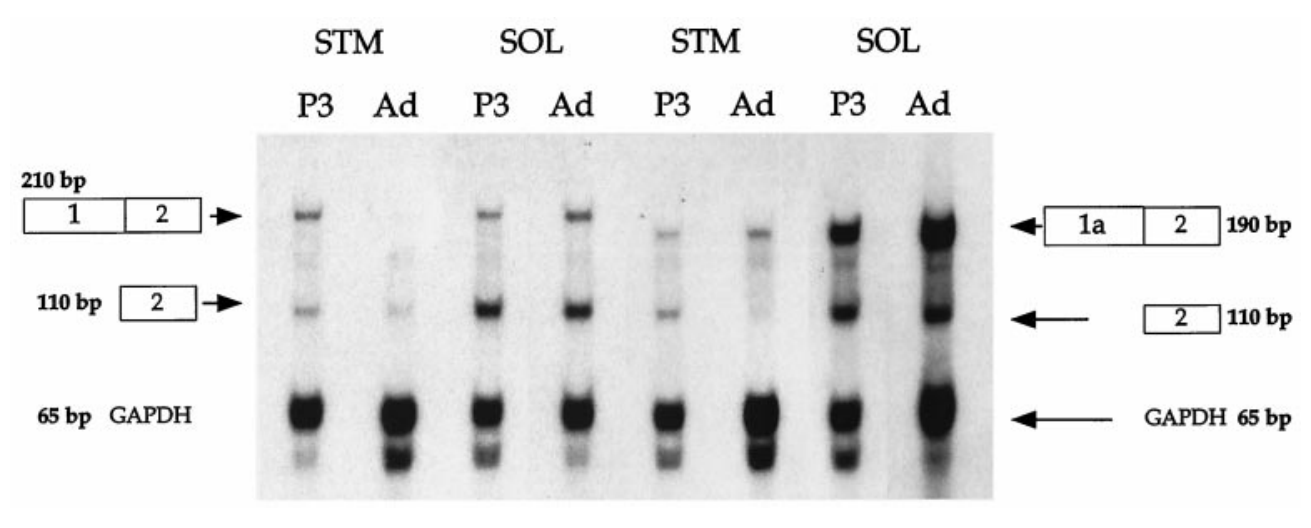

In the present work, we show that the different patterns of AChE A forms, as observed in muscle extracts, can be explained by assuming that their collagen tails are homotrimeric, containing three PRADs, and that the degree of ColQ occupancy by $\mathrm{AChE}_{\mathrm{T}}$ tetramers reflects the relative proportions of catalytic and collagenous subunits. This is supported by a comparison of the levels of $\mathrm{AChE}_{\mathrm{T}}$ and ColQ mRNAs in extrajunctional versus junctional domains of the rat STM and soleus muscles. The presence of $\mathrm{A}_{8}$ and $\mathrm{A}_{4}$ forms in extrajunctional segments of the soleus corresponds to a higher abundance of ColQ mRNAs relative to $\mathrm{AChE}_{\mathrm{T}}$ mRNAs. The reverse is true in the junctional region of the STM, which only contains the $\mathrm{A}_{12}$ form. Trying to test this hypothesis more directly, we were able to mimic the observed proportions of $\mathrm{A}_{4}, \mathrm{~A}_{8}$, and $\mathrm{A}_{12}$ forms by coinjecting adequate amounts of $\mathrm{AChE}_{\mathrm{T}}$ and ColQ mRNAs in Xenopus oocytes.

We show that the expression of ColQ transcripts is higher in the soleus muscle than in the fast STM muscle as early as postnatal day 3 when the pattern of neural activation in fast and slow muscles is not yet perceptibly different (Navarrete and Vrbová, 1983). Moreover, the level of collagen-tailed AChE forms is much higher in the soleus muscle, after either prolonged denervation or transplantation and regeneration in the place of a fast muscle, than in the correspondingly treated fast muscle (Sketelj et al., 1992; Dolenc et al., 1994). Thus, it seems that slow muscle fibers of type 1 possess a greater capacity to express ColQ transcripts.

The fact that $\mathrm{AChE}_{\mathrm{T}}$ subunits are expressed in excess at the neuromuscular junctions of fast muscles explains how exercise can modulate the production of the membrane-bound $\mathrm{G}_{4}{ }^{\mathrm{a}}$ form, without affecting other AChE forms (Fernandez and Donoso, 1988; Jasmin and Gisiger, 1990); the level of this molecule probably depends on the amount of the hydrophobic anchor subunit, 




Figure 7. The pattern of collagen-tailed $\mathrm{A}_{12}, \mathrm{~A}_{8}$, and $\mathrm{A}_{4}$ forms is determined by the relative amount of ColQ1 and ColQ1a mRNAs. Xenopus oocytes were injected with a fixed amount of $\mathrm{AChE}_{\mathrm{T}}$ mRNA (2 ng/oocyte) together with $0.3\left(A, A^{\prime}\right), 0.15\left(B, B^{\prime}\right)$, and $0.07\left(C, C^{\prime}\right) \mathrm{ng}$ of ColQ1a mRNA (left: $A, B, C$ ) or ColQ1 mRNA (right: $\left.A^{\prime}, B^{\prime}, C^{\prime}\right)$. We obtained similar patterns of molecular forms with approximately twofold to threefold more ColQ1 mRNA than ColQ1a mRNA. At appropriate concentrations, both transcripts could generate either $\mathrm{A}_{12}$ or incomplete A forms.

which may recruit catalytic subunits without interfering with the assembly of the $\mathrm{A}_{12}$ collagen-tailed molecule, in case of $\mathrm{AChE}_{\mathrm{T}}$ surplus.

\section{The alternative transcription sites 1 and 1 a produce $\mathrm{N}$-terminal variants of ColQ that differ in their capacity to recruit $\mathrm{AChE}_{\mathrm{T}}$ subunits}

An analysis of ColQ transcripts revealed the existence of two transcription initiation sites, so that transcripts start with either exon 1 or exon 1a. Both exons encode hydrophobic N-terminal regions that correspond mostly to putative signal peptides. Transcript 1 corresponds to the ColQ cDNA described previously (Krejci et al., 1997). Two similar alternative transcripts corresponding to ColQ1 and ColQ1a have also been isolated from human muscle (Ohno et al., 1998), suggesting that their existence may have a functional significance.

The two types of mRNAs should produce secreted ColQ subunits differing only by a few residues at the $\mathrm{N}$ terminus of the mature protein, i.e., upstream of the PRAD domain. Deletion experiments suggest that the peptide sequence preceding the PRAD is unlikely to influence the binding of $\mathrm{AChE}_{\mathrm{T}}$ subunits
(Bon et al., 1997), so that the two ColQ variants should be functionally equivalent. However, coexpression with $\mathrm{AChE}_{\mathrm{T}}$ in Xenopus oocytes revealed that the ColQ1a transcript is more efficient than ColQ1 for recruiting $\mathrm{AChE}_{\mathrm{T}}$ tetramers. This functional difference could result from the production of collagen subunits, because of the stability of the transcripts, the efficiency of their translation, or the stability of the resulting protein. A more interesting hypothesis is that both transcripts yield the same amounts of equivalent collagen subunits, but that the different signal peptides target them to different subdomains of the endoplasmic reticulum in which they assemble more or less efficiently with $\mathrm{AChE}_{\mathrm{T}}$ subunits.

The tissue expression of ColQ1 and ColQ1a is not identical. ColQ1 appears less restricted to muscle junctions, because it also occurs in the lung (data not shown), in the cardiac muscle, and in extrajunctional regions of the soleus.

\section{Synaptic localization of AChE collagen-tailed forms}

How can we explain that the ColQ protein is accumulated at neuromuscular junctions, although its mRNA is uniformly expressed along the soleus muscle? This question does not apply only to the rat soleus, but more generally to muscles in which collagen-tailed forms are extracted from nonjunctional, as well as junctional, regions, such as the rat immature muscles (Bevan and Steinbach, 1977; Sketelj and Brzin, 1980) and human or primate muscles (Carson et al., 1979; Sketelj and Brzin, 1985). The integration of collagen-tailed AChE in the synaptic basal lamina requires multiple steps, each of which can contribute to its local accumulation.

First, the complete $\mathrm{A}_{12}$ form could be the only secreted mature oligomer. $\mathrm{AChE}_{\mathrm{T}}$ subunits possess a $\mathrm{C}$-terminal peptide, the tryptophan amphiphilic tetramerization (WAT) domain, that serves as a tetramerization domain, interacting with the PRAD of ColQ (Simon et al., 1998). It is conceivable that the two interaction partners, PRAD and WAT, might behave as signals for retention and/or degradation when they are not associated. In this case, $\mathrm{AChE}_{\mathrm{T}}$ subunits containing an exposed WAT, as well as $\mathrm{A}_{4}$ and $\mathrm{A}_{8}$ forms containing an unoccupied PRAD, would not be externalized. In both fast and slow muscles, the junctional accumulation of $\mathrm{AChE}$ would then result from the fact that $\mathrm{A}_{12}$ is preferentially produced at this site because of the high local expression of $\mathrm{AChE}_{\mathrm{T}}$ subunits. It has indeed been shown that junctional nuclei of the rat soleus preferentially produce the $A_{12}$ form, like the junctional nuclei of the STM muscle (Sketelj et al., 1998).

Second, the junctional localization of collagen-tailed AChE may reflect the presence of synaptic binding sites. Selective localization of binding sites for collagen-tailed $\mathrm{AChE}$ in the junctional basal lamina was indeed demonstrated by attachment of quail A forms to frog neuromuscular junctions (Rotundo et al., 1997). The integration of collagen-tailed AChE in the synaptic basal lamina results from sequential steps that implies ionic interactions, followed by covalent attachment to the matrix (Rossi and Rotundo, 1996).

The collagen tail interacts with heparan sulfated proteoglycans (Bon et al., 1978; Vigny et al., 1983; Brandan and Inestrosa, 1984; Brandan et al., 1985), probably through heparin binding sites located in the triple-helical domain (Deprez and Inestrosa, 1995). Such interactions may contribute to the anchoring of collagentailed AChE at the neuromuscular junction at which heparan sulfate proteoglycan itself is concentrated (Anderson and Fambrough, 1983; Hall and Sanes, 1993). It has been shown recently 
that perlecan, which associates with the synaptic complex of dystroglycans, may constitute an acceptor site for collagen-tailed AChE (Peng et al., 1999). However, heparin can block initial interactions of newly synthesized A forms with the extracellular matrix but does not detach pre-existing clusters of A forms from the synaptic basal lamina (Rossi and Rotundo, 1993, 1996).

The members of a family affected by a mild form of CMS-1c, characterized by the absence of $\mathrm{AChE}$ at the neuromuscular junction, produced normal level of $\mathrm{A}$ forms that aggregate with heparan sulfate. The genetic analysis revealed a point mutation in the C-terminal, noncollagenous region of the ColQ subunit (Donger et al., 1998). Thus, the functional anchoring of the collagen-tailed enzyme requires its interaction with an as yet unidentified receptor site. It is likely that these sites are exclusively junctional, explaining the specific accumulation of collagentailed AChE at the neuromuscular junctions.

The covalently attached fraction of collagen-tailed AChE may represent the functional, and perhaps the major, component of these molecular forms, which is detected by immunofluorescence. The sedimentation patterns of muscle extracts do not allow an analysis of this unextractible component but only characterize the soluble component that probably represents recently synthesized molecules, the composition of which is explained by the ratio of $\mathrm{AChE}_{\mathrm{T}}$ and ColQ subunits.

Most postsynaptic proteins involved in neuromuscular transmission are expressed locally (Moscoso et al., 1996); in some, such as $\mathrm{S}$ laminin, post-translational processes can also contribute to synaptic localization (Martin et al., 1995). The ColQ gene represents an original case, because its transcripts are restricted to the end plates only in fast rat muscles but are uniformly distributed in the slow soleus muscle, although the protein is concentrated at the junctions in both muscles. It is intriguing that the mechanisms that govern the accumulation of synaptic components, such as ColQ and AChE, depend on the type of muscle fiber.

\section{REFERENCES}

Anderson MJ, Fambrough D (1983) Aggregates of acetylcholine receptors are associated with plaques of basal lamina heparan sulfate proteoglycan on the surface of skeletal muscle fibers. J Cell Biol 97:1396-1411.

Bevan S, Steinbach JH (1977) The distribution of alpha-bungarotoxin binding sites of mammalian skeletal muscle developing in vivo. J Physiol (London) 267:195-213.

Bon S, Massoulié J (1997) Quaternary associations of acetylcholinesterase. I. Oligomeric associations of $\mathrm{T}$ subunits with and without the amino-terminal domain of the collagen tail. $\mathrm{J}$ Biol Chem 272:3007-3015.

Bon S, Cartaud J, Massoulié J (1978) The dependence of acetylcholinesterase aggregation at low ionic strength upon a polyanionic component. Eur J Biochem 85:1-14.

Bon S, Coussen F, Massoulié J (1997) Quaternary associations of acetylcholinesterase. II. The polyproline attachment domain of the collagen tail. J Biol Chem 272:3016-3021.

Brandan E, Inestrosa NC (1984) Binding of asymmetric forms of acetylcholinesterase to heparin. Biochem J 221:415-422.

Brandan E, Maldonado M, Garrido J, Inestrosa NC (1985) Anchorage of collagen-tailed acetylcholinesterase to the extracellular matrix is mediated by heparan sulfate proteoglycans. J Cell Biol 101:985-992.

Carson S, Bon S, Vigny M, Massoulié J, Fardeau M (1979) Distribution of acetylcholinesterase molecular forms in neural and non-neural sections of human muscle. FEBS Lett 97:348-352.

Č́resnar B, Č́rne-Finderle N, Breskvar K, Sketelj J(1994) Neural regulation of muscle acetylcholinesterase is exerted on the level of its mRNA. J Neurosci Res 38:294-299.

Chomczynski P, Sacchi N (1987) Single-step method of RNA isolation by acidic guanidium thiocyanate-phenol-chloroform extraction. Anal Biochem 162:156-159.
Deprez P, Inestrosa NC (1995) Two heparin-binding domains are present on the collagenic tail of asymmetric acetylcholinesterase. J Biol Chem 270:11043-11046.

Dolenc I, Cérne-Finderle N, Erzen I, Sketelj J (1994) Satellite cells in slow and fast rat muscles differ in respect to acetylcholinesterase regulation mechanisms they convey to their descendant myofibers during regeneration. J Neurosci Res 37:236-246.

Donger C, Krejci E, Pou Serradell A, Eymard B, Bon S, Nicole S, Chateau D, Gary F, Fardeau M, Massoulié J, Guicheney P (1998) Mutation in the human acetylcholinesterase-associated collagen gene, $\mathrm{ColQ}$, is responsible for congenital myasthenic syndrome with endplate acetylcholinesterase deficiency (type Ic). Am J Hum Genet 63:967-975.

Ellman GL, Courtney KD, Andres V, Featherstone RM (1961) A new and rapid colorimetric determination of acetylcholinesterase activity. Biochem Pharmacol 7:88-95.

Feng G, Krejci E, Molgo J, Cunningham JM, Massoulié J, Sanes JR (1999) Genetic analysis of Collagen Q: roles in acetylcholinesterase and butyrylcholinesterase assembly and in synaptic structure and function. J Cell Biol 144:1349-1360.

Fernandez HL, Donoso JA (1988) Exercise selectively increases G4 AChE activity in fast-twitch muscle. J Appl Physiol 65:2245-2252.

Fernandez HL, Seiter TC (1984) Subcellular distribution of acetylcholinesterase asymmetric forms during postnatal development of mammalian skeletal muscle. FEBS Lett 170:147-151.

Hall ZW (1973) Multiple forms of acetylcholinesterase and their distribution in endplate and non-endplate regions of rat diaphragm muscle. J Neurol 4:343-361.

Hall ZW, Kelly RB (1971) Enzymatic detachment of endplate acetylcholinesterase from muscle. Nat New Biol 232:62-64.

Hall ZW, Sanes JR (1993) Synaptic structure and development: the neuromuscular junction. Cell 72:99-121.

Jasmin BJ, Gisiger V (1990) Regulation by exercise of the pool of G4 acetylcholinesterase characterizing fast muscles: opposite effects of running training in antagonist muscles. J Neurosci 10:1444-1454.

Koelle GB, Friendenwald JS (1949) A histochemical method for localizing cholinesterase activity. Proc Soc Exp Biol 70:617-622.

Koenig J, Rieger F (1981) Biochemical stability of the AChE molecular forms after cytochemical staining: postnatal focalization of the $16 \mathrm{~S}$ AChE in rat muscle. Dev Neurosci 4:249-257.

Krejci E, Coussen F, Duval N, Chatel JM, Legay C, Puype M, Vandekerckhove J, Cartaud J, Bon S, Massoulié J (1991) Primary structure of a collagenic tail peptide of Torpedo acetylcholinesterase: coexpression with catalytic subunit induces the production of collagentailed forms in transfected cells. EMBO J 10:1285-1293.

Krejci E, Thomine S, Boschetti N, Legay C, Sketelj J, Massoulié J (1997) The mammalian gene of acetylcholinesterase-associated collagen. J Biol Chem 272:22840-22847.

Lai J, Jedrzejczyk J, Pizzey JA, Green D, Barnard EA (1986) Neural control of the forms of acetylcholinesterase in slow mammalian muscles. Nature 321:72-74.

Legay C, Huchet M, Massoulié J, Changeux JP (1995) Developmental regulation of acetylcholinesterase transcripts in the mouse diaphragm: alternative splicing and focalization. Eur J Neurosci 7:1803-1809.

Lømo T, Massoulié J, Vigny M (1985) Stimulation of denervated rat soleus muscle with fast and slow activity patterns induces different expression of acetylcholinesterase molecular forms. J Neurosci 5:1180-1187.

Marsh D, Grassi J, Vigny M, Massoulié J (1984) An immunological study of rat acetylcholinesterase: comparison with acetylcholinesterases from other vertebrates. J Neurochem 43:204-213.

Martin PT, Ettinger AJ, Sanes JR (1995) A synaptic localization domain in the synaptic cleft protein laminin beta 2 (s-laminin). Science 269:413-416.

Massoulié J, Sussman J, Bon S, Silman I (1993) Structure and functions of acetylcholinesterase and butyrylcholinesterase. Prog Brain Res 98:139-146.

Moscoso L, Merlie JP, Sanes JR (1996) N-CAM, 43K-rapsyn, and s-laminin mRNAs are concentrated at synaptic sites in muscle fibers. Mol Cell Neurosci 6:80-89.

Navarrete R, Vrbová G (1983) Changes of activity patterns in slow and fast muscles during postnatal development. Dev Brain Res 8:11-19.

Ohno K, Brengman J, Tsujino A, Engel AG (1998) Human endplate acetylcholinesterase deficiency caused by mutations in the collagen-like 
(ColQ) of the asymmetric enzyme. Proc Natl Acad Sci USA 95:9654-9659.

Peng HB, Xie H, Rossi SG, Rotundo RL (1999) Acetylcholinesterase clustering at the neuromuscular junction involves perlecan and dystroglycan. J Cell Biol 145:911-921.

Rossi SG, Rotundo RL (1993) Localization of "non-extractible" acetylcholinesterase to the vertebrate neuromuscular junctions. J Biol Chem 268:19152-19159.

Rossi SG, Rotundo RL (1996) Transient interactions between collagentailed acetylcholinesterase and sulfated proteoglycans prior to immobilization ion the extracellular matrix. J Biol Chem 271:1979-1987.

Rotundo RL, Rossi SG, Anglister L (1997) Transplantation of quail collagen-tailed acetylcholinesterase molecules onto the frog neuromuscular synapse. J Cell Biol 136:367-374.

Simon S, Krejci E, Massoulié J (1998) A four-to-one association between peptide motifs: four C-terminal domains from cholinesterase assemble with one proline-rich attachment domain (PRAD) in the secretory pathway. EMBO J 17:6178-6187.

Sketelj J, Brzin M (1979) Attachment of acetylcholinesterase to structures of the motor endplate. Histochemistry 61:239-248.
Sketelj J, Brzin M (1980) $16 \mathrm{~S}$ acetylcholinesterase in endplate-free regions of developing rat diaphragm. Neurochem Res 5:653-658.

Sketelj J, Brzin M (1985) Asymmetric molecular forms of acetylcholinesterase in mammalian skeletal muscles. J Neurosci Res 14:95-103.

Sketelj J, Čérne-Finderle N, Brzin M (1992) Influence of denervation on the molecular forms of junctional and extrajunctional acetylcholinesterase in fast and slow muscles of the rat. Neurochem Int 21:415-421.

Sketelj J, Č́rne-Finderle N, Ítrukelj JV, Trontelj JV, Pette D (1998) Acetylcholinestrase mRNA level and synaptic activity in rat muscles depend on nerve-induced pattern of muscle activation. J Neurosci 18:1944-1952.

Toutant JP, Massoulié J (1988) Cholinesterases: tissue and cellular distribution of molecular forms and their physiological regulation. Handb Exp Pharmacol 86:225-265.

Vigny M, Koenig J, Rieger F (1976) The motor end-plate specific form of acetylcholinesterase: appearance during embryogenesis and reinnervation of rat muscle. J Neurochem 27:1347-1353.

Vigny M, Martin GR, Grotendorst GR (1983) Interactions of asymmetric forms of acetylcholinesterase with basement membrane components. J Biol Chem 258:8794-8798. 